\title{
KOMMUNIKATIVE UND KOGNITIVE FUNKTIONEN VON METAPHERN IM PUBLIZISTISCHEN TEXT
}

\author{
Lekt. Jurgita Lukienè \\ Kaunas technologische Universität, \\ Doc. dr. Nijolė Čiučiulkienė \\ Aleksandras Stulginskis Universität \\ Dainoras Lukas \\ Journalist, Soziologe
}

\begin{abstract}
Annotation. In diesem Artikel werden kommunikative und kognitive Metaphernfunktionen in der Textsorte Interview analysiert. Die wichtigste Frage des Themas - was gibt uns die Metapher? Die Funktion dieser Stilfiguren wurde in verschiedenen Zeitabschnitten unterschiedlich verstanden. Heute wird die Metapher sehr breit benutzt. Man trifft sie nicht nur in der Literatur, sondern auch in der technischen Sprache, Presse. Das Ziel des Sprechers ist nicht nur eine bestimmte Metaphernbedeutung, sondern auch ihre Funktion. Sie hilft etwas Neues zu sehen und kennenzulernen. Die moderne Sprache widerspiegelt sich am besten in der Presse. Wichtig sind hier die Wortwahl, Satzstruktur, das Thema, die Situation. Deswegen wurde im praktischen Teil die politische und wirtschaftliche Zeitschrift „Der Spiegel“ analysiert. Die Themen sind breit-Geld, Wahl, Umweltschutz, Arbeit, Ausland, Reformen, Menschenrechte, Welt, Finanzen, Elektronik, Autos, Mechanik, Medien. Von verschiedenen Interviews haben wir insgesamt 309 Metaphernbeispiele gesammelt. Die Beispiele wurden nach lexikologischen und kommunikativen Merkmalen analysiert. Das Ziel des Artikels ist nicht nur das Verstehen einer Metapher, sondern auch ihre Funktion im Text, deswegen ist in unserer Untersuchung sowohl das Ziel des Sprechers, als auch die Wirkung der Metapher von großer Wichtigkeit. Es ist zu merken, dass die Metaphern in der Sprache der Politiker und Wirtschaftsleute sehr informativ und effektiv sind. In ihrer Sprache widerspiegelt sich die Einschätzung und übertriebene Situation. Die Wirkung von Metaphern ist unterschiedlich, oft können sie nur aus dem Kontext verstanden werden. In den analysierten Beispielen findet man am meisten Kriegs-, Tier-, Sport- und Theatermetaphern.
\end{abstract}

Schlüsselwörter: Metapher, Politik und Wirtschaft, Interview. 


\section{Einleitung}

Seit dem Altertum wurde gedacht, dass Metaphern nur für rhetorische Zwecke dienen. Heute werden sie nicht nur in der Literatur, sondern auch in der Alltagssprache gebraucht. Mit dem Thema beschäftigen sich verschiedenen Wissenschaften: Literatur und Sprachwissenschaft, Philosophie, Soziologie u andere Wissenschaften. Die Metapher taugt zum Lob und Tadel, zum Informieren u Verbergen, zum Beschreiben und zur Wahrnehmung. Eine der wichtigsten Thesen des Artikels ist, dass die Metapher als eine Form der Verständigung neben der gewöhnlichen Kommunikation zu verstehen ist. Soziale Wirklichkeit wird durch die Art und Weise der Sprache konstruiert, d.h., wie über Sachverhalte gesprochen wird - etwa durch Metaphern. Metaphern machen unsere Sprache reich.

Problem. Heutzutage wird die soziale Wirklichkeit durch moderne Medien dargestellt. In den Medien erwarten die Nutzer/Leser einen expressiven Diskurs. Deswegen werden für die Publizistik Stil eine Menge Stilanforderungen gestellt. Er muss nicht nur logisch und genau, sondern auch eindrucksvoll, emotional und bildhaft sein (vgl. Karaliūnas, 1997). Die Frage ist, ob die heutige Presse die erwähnten Kriterien entspricht.

Erforschungsobjekt: Metaphernanalyse.

Ziel der Untersuchung ist es, am Beispiel der politischen und wirtschaftlichen Interviews in der Zeitschrift „Der Spiegel“ das bildhafte Sprechen der Politiker und Wirtschaftsleute durch die kommunikativen und kognitiven Funktionen von Metaphern zu zeigen

Um das Ziel zu erreichen, nehmen wir folgende Aufgaben vor:

1. Die wichtigsten Theorien der Metapher zu analysieren

2. Durch semantische Definition und Interpretation die Frage zu beantworten, was die Metapher kommuniziert.

3. In den politischen und wirtschaftlichen Interviews in der Zeitschrift

„Der Spiegel“" kommunikative und kognitive Funktionen zu analysieren.

In diesem Artikel werden folgende Forschungsmethoden verwendet:

1. Analyse der wissenschaftlichen Literatur

2. Grammatische und semantische Inhaltsanalyse der Metaphern.

Der Artikel besteht aus einem theoretischen und empirischen Teil. Im theoretischen Teil werden die wissenschaftlichen Quellen analysiert und die wichtigsten Theorien beschrieben; der empirische Teil umfasst die linguistische Analyse der Metapher in den Interviewtexten aus der Zeitschrift „Der Spiegel“. Die Beispiele werden zuerst nach den Wortarten Substantiv, Adjektiv, Verb klassifiziert, dann nach Grundthemen sortiert und semantisch definiert und interpretiert. In dem dritten Teil werden Forschungsergebnisse beschrieben und die Schlussfolgerungen werden am Ende des Artikels vorgestellt. 


\section{Theoretische Analyse der Metapher-Definition und der wichtigsten Metapherntheorien}

Die Metapher wird traditionell als die wichtigste der rhetorischen Figuren betrachtet. Das Wort „Metapher“ geht etymologisch auf Griechisch „metaphora“ zurück. „Metapherein“ heißt ,anderswohintragen“. Im Duden Universalwörterbuch (1999) ist die Metapher, ,sprachlicher Ausdruck, bei dem ein Wort (eine Wortgruppe) aus seinem Bedeutungszusammenhang in einem anderen übertragen, als Bild verwendet wird“.

Die Metapher, die in der Antike ,als verkürzter Vergleich“, als Verbindung zweier ähnlicher Bildfelder ohne Vergleichspartikel gekennzeichnet worden war, hat in der linguistischen Strukturforschung und Stilistik bisher die meiste Aufmerksamkeit von allen Tropen und Figuren gezogen, so dass die Metaphernforschung heute einen eigenen Spezialforschungsbereich ausmacht" (Sowinski,1991, 2007)

In der antiken Rhetorik erklärt man die Metapher als Hinübertragung eines fremden Wortes. Hier gilt die Metapher als „Schmuck“ der Rede. Sie wirkt bei der Meinungsbeeinflussung und gestaltet die Sprache effektvoll. Deswegen spielt die metaphorische Redeweise eine wichtige Rolle im Altertum: sie spielt eine zentrale Rolle in der Gerichts- und Festrede. In der rationalistischen Philosophie des 17. Jhs. interpretiert man die Metapher nur als stilistisches Mittel und wertet den metaphorischen Sprachgebrauch in der Philosophie und Wissenschaft ab. Im Mittelalter erweckt die „uneigentliche“ Rede den Menschen kein großes Vertrauen. Eine einflussreiche Arbeit zur Metaphorologie hat Ivor Armstrong Richards geleistet. Er beginnt damit, dass die Wörter der Bedeutungen nicht stabil sind und sie sich von Kontext zu Kontext verändern. Max Black erweitert Richards Theorie. Sein Gesichtspunkt ist, dass die Metaphern uns erlauben „einen Begriff aus der Perspektive eines anderen zu sehen“ (Frieling, 1996).

Harald Weinrich beschäftigt sich mit der Thematik der Metapher unter mehreren Aspekten. Er bezieht sich in seiner Auffassung zur Lehre der kühnen Metapher auf die Antike und deren Vertreter. Die Metapher wird mit menschlichen Charakteristika wie auch mithilfe der anderen Metaphern beschrieben. Die Metaphorik lebt von Distanz. Die Distanz wird Spanne, Abstand zwischen Bild und Sache genannt. Der Forscher meint, je weiter die Bildspanne ist, umso kühner gelingt die Metapher (vgl. J.Nieraad, 1997). Wenn der Abstand zu groß ist, funktioniert die Metapher nicht. Die Metaphorik entsteht, indem „wörtliche“ und „metaphorische“ Bedeutungen in Verbindung gebracht werden. Eine Metapher ist mehr als ein Wort im Kontext, sie bekommt im Text eine unerwartete Bedeutung. Metaphern sind nach Weinrich wichtig, praktisch, ohne ein Schmuckelement und die moderne Sprache enthält viele Metaphern.

Erwähnenswert sind auch die Arbeiten zur Metaphernforschung von Lakoff und Johnson. Sie formulieren ihre Theorie im Rahmen der kognitiven Linguistik. 
Ihr Gedanke ist, dass Metaphern nicht nur bloßes Dekor der Sprache sind, sondern dass wir sie überall gebrauchen und dass unser gesamtes Konzeptsystem von ihnen geprägt ist. Die metaphorische Denkweise liegt schon in der Kultur der Menschen, nach der auch unser Handeln zu verstehen ist.

Laut Katrin Kohl erzeugen Metaphern eine >bildliche< Rede, in der Wörter nicht ihre >eigentliche`, >wörtliche< Bedeutung vermitteln, sondern eine (meist abstraktere) >uneigentlicheく, >übertragene`, >metaphorischeく Bedeutung. (vgl. Kohl, 2007).

\subsection{Theorien der Metaphern}

Die Klassifikation der Metaphern erfolgt nach den drei Haupttheorien. Die Substitutions- bzw. Ersetzungstheorie ist die älteste und geht auf Aristoteles zurück. Laut dieser Theorie wird bei der Metapher der wörtliche Ausdruck durch einen fremden ersetzt. Die Metapher gilt in der rhetorischen Tradition als Schmuck und Mittel der poetischen Redeweise. Diese Einstellung hat sich im Laufe der Zeit verändert, weil nicht übersehen werden konnte, dass auch die alltägliche Rede voll von Metaphern ist. Eine Variante der Substitutionstheorie ist die Vergleichstheorie. Dieser Theorie zufolge ist die Metapher ein verkürzter Vergleich, der Metapher fehlt nur die Vergleichspartikel „wie“. Der Begriff der Ähnlichkeit ist unter bestimmtem Gesichtspunkt problematisch, weil die Dinge nur in bestimmten Hinsichten ähnlich sind. Die Metapher ist kein Vergleicht nicht nur deswegen, weil die Vergleichspartikel fehlt. Die Metapher kann man erläutern, in dem man sie umschreibt. Diese Theorie wurde von vielen Forschern kritisiert, weil die Leistung der Metapher nur als verkürzter Vergleich betrachtet wird. Die stilistische Funktion sei demzufolge, den Hörer zu erfreuen und die Sprache zu schmücken. Andererseits hatte diese Theorie großen Einfluss auf die nachfolgenden Arbeiten zur Metaphorik.

Die Rolle der Metapherändert sich innerhalb der Sprache. Diese Figurwirdnicht mehr als Schmuck betrachtet und kann nicht auf eine wörtliche Bedeutung reduziert werden. Max Black erweitert diese Ideen in der Interaktionstheorie. „Interaktion“ bedeutet Wechselbeziehung zwischen Handlungspartnern. Die Interaktionstheorie basiert auf der Betrachtung der einzelnen sprachlichen Erscheinung, also auch der Metapher, als Bestandteil der ganzen Kommunikationssituation. Diese Theorie untersucht, ob ein Ausdruck in der Gesamtheit einer Äußerung als Metaphorisch gilt. Die Bedeutung eines Wortes hängt davon ab, wie es verwendet wird, d.h. derselbe Ausdruck kann je nach Kontext metaphorisch sein oder nicht. M. Black konstatiert, dass Metaphern nicht nur als stilistische Dekorationen dienen. Sie wirken auf die Gefühle des Lesers, helfen neue Dinge zu begreifen. Wir können durch die Metaphern einen Begriff aus der Perspektive des anderen sehen.

Die Betrachtung der Metaphern durch Lakoff und Johnson eröffnet einen neuen Blickwinkel der Metaphernforschung. Ihr Ziel ist, das Auftreten von Metaphern in den Sprachkontexten im Hinblick auf kognitive Prozesse zu interpretieren. Wir 
können durch Metaphern Unsagbares formulieren, tiefe Gedanken ausdrücken. Die Forscher sind der Ansicht, dass die Menschen die Metaphern ständig gebrauchen, um verbal kommunizieren zu können. Sie beeinflussen nicht nur unsere Sprache, sondern auch unser Denken und Handeln. Auf diese Erkenntnis aufbauend, kommen Lakoff und Johnson zu dem Schluss, dass unser gesamtes Konzeptsystem von Metaphern geprägt ist. So verdeutlichen sie, dass die menschliche Denkweise und auch unser Handeln auf einer metaphorischen Grundordnung basiert.

\subsection{Kommunikative Funktion der Metapher}

Kommunizieren lässt sich als besondere Form des Verhaltens bestimmen. Jede menschliche Äußerung hat bestimmte Intentionen oder Gründe. Es ist für die Kommunikation auch wichtig, wie die Sprache gebraucht wird und welche Wirkung sie hat.

Im Duden Wörterbuch wird das Wort „kommunikativ“ als „1. die Kommunikation betreffend; mitteilsam“ erklärt. Unter „Kommunikation“ (lat. communicatio) versteht man Verbindung, Mitteilung. Aus diesen Definitionen ergibt sich die spezifische Leistung der Metapher. „Während unter dem Begriff der kognitiven Funktion die Relevanz von Metaphern für das Verstehen von Ereignissen etc. gefasst wurde, meint kommunikative Funktion die Relevanz für konkrete Kommunikationssituationen und darüber hinaus für kommunikative Prozesse in einer Sprachgemeinschaft“" (Kirchhoff, 2015).

Wenn wir sprechen, wählen wir bestimmte Zeichen aus unserem mentalen Lexikon aus und verknüpfen sie mit anderen Zeichen. Durch diese Verknüpfung werden die Wörter zu Metaphern. Solche Verkettung wirkt ungewöhnlich, überraschend und phantasievoll. Sie gibt eine Perspektive, neue Eigenschaften am betrachteten Gegenstand zu sehen. Die Wirksamkeit der Metapher besteht darin, dass ,sie neue Aspekte einer Sache oder intensive Konnotationen einer Rede, eines bezeichneten Menschen, Gegenstandes oder Ablaufs erlaubt“"(Glück, 2016). Mit der metaphorischen Äußerung werden bestimmte Intensionen und Interessen formuliert. Die Sprechenden haben im Kontext das Neue, Unerwartete, das die Situation bildlicher, anschaulicher und zugleich informativer macht. Einen ausdrucksreichen Effekt haben die Metaphern in den politischen und wirtschaftlichen Interviews. Z.B.: im Wirtschaftsbereich - „Murdochs größter Einzelaktionär ist der US-Kabelgigant John Malone“ (Der Spiegel Nr. 9/25.2.02, S.98).

Aus diesem Beispiel lässt sich bestimmen, dass die Metapher etwas veranschaulichen, zur Ironie beitragen, hervorheben, belügen, bewerten kann.

Metapher kann man als eine Äußerung betrachten, die nicht als isolierte Erscheinung zu betrachten ist. Mit einer Äußerung wird eine kommunikative Situation gegeben und die Sprachliche Bedeutung ergibt sich nur in bestimmter Situation, für bestimmte Sprecher und Hörer. Es gibt Metapher, die man nicht verstehen kann, ohne die Persönlichkeit zu kennen, z.B.: „Ist Putin dabei aktiver 
Inhaber der Macht oder bloße Marionette?" (Der Spiegel Nr. 2/10.1.00, S.114) Um diese Metapher verstehen zu können, brauchen wir politisches Wissen und warum, zu welchem Zweck die Person „bloße Marionette“ genannt wird. Wir beherrschen Regeln und Normen des Kommunizierens und können den kommunikativmetaphorischen Austausch zu unseren Zwecken einsetzen. Was in kommunikativer Absicht gesagt wird, hat einen Zweck. Ohne Metaphern wäre unsere Sprache statisch. Das gilt nicht nur für die Alltagskommunikation, sondern auch für die Literatur-, Pressesprache.

\subsection{Kognitive Funktion der Metapher}

Die Beschäftigung mit der kognitiven Funktion der Sprache beginnt zusammen mit der Entstehung der kognitiven Linguistik, die in der 2. Hälfte des 20. Jahrhunderts entstanden ist. „Die Metapher hat einen kognitiven Aspekt (kognitive Metapher, konzeptuelles Modell) und einen kommunikativ sprachlichen Aspekt (metaphorischer Ausdruck). Diese Aspekte wirken interaktiv bzw. als Ganzheit. Aus mentaler Perspektive ist die Metapher das Ergebnis einer >Projektion`von Elementen einer kognitiv-sprachlichen Einheit (aus einem meist konkreten $>$ Herkunftsbereich $\iota$ ) auf eine andere kognitivsprachliche Einheit (einen meist abstrakteren >Zielbereich $<$ )“(Kohl, 2007).

Die kognitive Linguistik untersucht das Zusammenwirken von Sprache und mentaler Tätigkeit des Menschen (seiner Denk- und Erkenntnistätigkeit). Ihr „Untersuchungsziel ist die Erforschung der kognitiven/mentalen Struktur und Organisation durch Analyse der kognitiven Strategien, die Menschen beim Denken, Speichern von Information, Verstehen und Produzieren von Sprache verfolgen“ (vgl. Bußmann \& Lauffer, 2008). Im Mittelpunkt dieser Disziplin steht die Sprache als kognitives Instrument, das die Kodierung und Verarbeitung von Information gewährleistet. Das Internet-Duden Wörterbuch beschreibt das Wort „kognitiv“" als „das Wahrnehmen, Denken, Erkennen betreffend“" (https://www. duden.de/rechtschreibung/kognitiv). Die Funktion des Wahrnehmens bestimmte schon Aristoteles, er behauptete, die Metapher führt zu einer „Wahrnehmung von Ähnlichkeiten“. D. Davidson interpretiert auch den kognitiven Inhalt der Metapher, er sagt, sie führt uns zu Erkenntnis von Dingen, die wir andernfalls nicht bemerken würden und dadurch beeinflusst die Metapher unsere Visionen, Gedanken, Gefühle, bringt überraschende Ähnlichkeiten zu Bewusstsein (vgl. Davidson, 1998)

Aus diesen Definitionen lässt sich die Bestimmung der kognitiven Funktion deuten. Man kann die Metapher als spezifische Weltauffassung, Weltansicht betrachten. Sie ermöglicht ein uneinheitliches Denken, ist sparsam und umfangreich zugleich. Der metaphorische Ausdruck aktiviert die Aufnahme des Gesagten durch den Hörer/Leser. Die Metapher rekonstruiert das konzeptuelle System. Der menschliche Geist konstruiert das Gesagte und ist auch in der Lage, es zu verändern. Für das Verständnis eines metaphorischen Satzes ist neben dem 
sprachlichen Wissen das Weltwissen notwendig (vgl. Frieling, 1996). Das Verstehen einer Metapher gleicht dem „Entziffern eines Codes“. Sie wird wirksam, indem sie erkannt, wahrgenommen wird.

Da die Metapher ein Mittel der indirekten Äußerung ist, der übertragenen Darstellung einer Erscheinung ist, dient sie als Quelle der kreativen Aussagekraft. Nach den Gesetzen der Wahrnehmungspsychologie beeindruckt eine indirekte Äußerung mehr als eine direkte, denn die indirekte Äußerung regt unsere Aufmerksamkeit und Phantasie an.

\section{Empirischer Teil}

In keinem anderen Medium wird die Gegenwartssprache so wiedergespiegelt wie in der Presse. Hier spielen Wortwahl, Satzbau, Thema, Situation eine wichtige Rolle. Die Intention der Pressesprache ist die informative Verpflichtung. Die Sprache variiert nach der sozialen Schicht, die angesprochen wird und nach der Intention soll der Text mehr informieren, meinungsbildend oder politisch-agitatorisch sein.

Interview ist eine Art Kommunikation. „Das Interview soll auf möglichst unterhaltsame Art nicht nur Wissen und Meinungen, sondern auch die Denkweise(n) bemerkenswerter oder für die Sache aufschlussreicher Personen als Abfolge von Äußerungen in einer authentischen Form zur Darstellung bringen“ (Haller, 2001). D.h., in einem Interview sind authentische Aussagen, Meinungen, bestimmte Redewendungen enthalten.

In der Zeitschrift „Der Spiegel“ bestehen Interviews aus Frage-AntwortSätzen. Der Interviewer formuliert meistens provokative Aussagen, die der Partner zu einer Gegenaussage veranlassen sollen. Da im Interview dem Befragten eine Gelegenheit gegeben wird, eigene Begründungen, Vorstellungen darzulegen, finden sich in dieser Textsorte größtenteils vollständige, vielfach umfangreiche Verbalsätze (vgl. Engel, 1991). In solchen Texten verwenden beide Partner stilistische Mittel wie Vergleiche, Metaphern, aufwertende Adjektive, die das Interesse des Rezipienten wecken und verstärken. Das Interview ergründet entweder die Haltung einer Person zu bestimmten Sachfragen oder stellt die Persönlichkeit eines Menschen dar. Das Ziel des Interviews ist zu informieren und zu überzeugen.

Die Gesprächspartner haben verschiedene Rollen: hinter der journalistischen Frage kann sehr viel Hintergrundinformation stecken; der Befragte kann die Antwort mit einer metaphorischen Äußerung sehr ausdrucksvoll und genau geben. D.h., im Interview widerspiegelt sich nicht nur der gesprochene Stil der Kommunikationspartner, sondern auch ihre Absichten, Stimmung und selbst die Persönlichkeit.

Für den empirischen Teil wurden zwei Grundthemen aus der Zeitschrift „Der Spiegel“" genommen: Politik und Wirtschaft. Diese Themen sind Breit, deswegen wurden sie noch in folgende Bereiche gegliedert: Politik - als Geld-, Wahlkampf-, Umwelt-, Arbeits-, Auslands-, Reformpolitik und Menschenrechte; Wirtschaft 
- als Welt-, Finanzenwirtschaft, Auto-, Medien- und Elektronikindustrie. Die metaphorischen Bespiele wurden zuerst nach semantischen Bereichen sortiert, um die Vielfältigkeit der Metaphern in diesem Feld zu zeigen.

Die Beispiele werden nach den kognitiv-semantischen Aspekten untersucht. Die Analyse wird aus der Sicht der Lexikologie gemacht. Die Wörter haben ihre direkte Bedeutung, die sich im Fall der Metaphorik ändert. Wenn man zwei Wörter aus verschiedenen Bereichen im bestimmten Kontext miteinander verbindet, erzeugen wir eine metaphorische Äußerung. Die Klassifikation erfolgt zuerst unter dem substantivischen, adjektivischen und verbalen Gesichtspunkt. Der zweite Schritt ist die semantische Beschreibung, d.h., das Definieren und Interpretieren. Von großer Relevanz ist kommunikative Kompetenz von Menschen. Es ist wichtig, wie sie die kommunikativen Intentionen verstehen und wie eine Metapher wirkt. Die Wirkung der Metaphern ist unterschiedlich und oft kann man sie nur aus dem Textzusammenhang erkennen. Die Metaphernverwendung beeindruckt jeden Leser stark und dient zu einem bestimmten Zweck. Die Metapher informiert, charakterisiert, drückt die Wertung aus. In jeder Gruppe wird ein typisches Beispielsmodell erläutert (siehe Tabelle 1.).

1. Tabelle. Grammatische und Semantische Analyse.

\begin{tabular}{|c|c|c|c|}
\hline Nr. & $\begin{array}{c}\text { Grammatische } \\
\text { Gruppe }\end{array}$ & Beispiel & Semantische Interpretation \\
\hline \multirow[t]{2}{*}{1.} & $\begin{array}{c}\text { Substantisvische } \\
\text { Gruppe }\end{array}$ & & \\
\hline & $\begin{array}{c}\text { Substantiv }+ \\
\text { Substantiv }\end{array}$ & $\begin{array}{l}\text { Der Schwarzgeld- } \\
\text { Jongleur (Prinz } \\
\text { Wittgenstein) }\end{array}$ & $\begin{array}{l}\text { Zirkusbereich. } \\
\text { Diese Metapher stammt aus der Geldpolitik und } \\
\text { wird mit dem Zirkusbereich verknüpft. Herr } \\
\text { Wittgenstein kann wahrscheinlich mit dem illegalem } \\
\text { Geld genauso gut umgehen, wie ein Jongleur im } \\
\text { Zirkus, indem er die Reifen in die Luft wirft und } \\
\text { mit großer Geschicklichkeit wieder auffängt. } \\
\text { Diese Metapher ironisiert, indem der Mann als } \\
\text { Zirkusakteur vorgestellt und bewertet wird. }\end{array}$ \\
\hline & $\frac{\text { Substantiv }}{\text { Substantiv }}+$ & $\begin{array}{l}\text { eine Ampel- } \\
\text { Koalition }\end{array}$ & $\begin{array}{l}\text { Verkehrsbereich. } \\
\text { Die Zusammensetzung entsteht aus der Verknüpfung } \\
\text { unterschiedlicher Lebensbereiche. „Ampel“ gehört } \\
\text { zu dem Verkehrsbereich und „Koalition“ stammt } \\
\text { aus der Politik. Statt direkt zu sagen, welche } \\
\text { Parteien die Koalition umfassen, nimmt man zur } \\
\text { Veranschaulichung ein gewöhnliches Phänomen } \\
\text { aus unserem Alltagsleben, d.h. die Ampel. Das } \\
\text { Wichtigste an diesem Wort ist die Farben rot, gelb } \\
\text { und grün. Sie sind Entsprechungen für bestimmte } \\
\text { Parteienfarben: Rot, Gelb und Grün oder SPD; } \\
\text { FDP und Die Grünen. Diese Metapher gilt auch } \\
\text { als ökonomischer Ausdruck und weckt die } \\
\text { Aufmerksamkeit. }\end{array}$ \\
\hline
\end{tabular}




\begin{tabular}{|c|c|c|c|}
\hline & $\frac{\text { Substantivische }}{\text { Gruppe }}$ & $\begin{array}{l}\text { der Spektakel der } \\
\text { letzten Wochen }\end{array}$ & $\begin{array}{l}\text { Theaterbereich. } \\
\text { Die Schwächen der politischen Konkurrenten } \\
\text { für alle zeigen. Diese substantivische Metapher } \\
\text { verein zwei Lebensbereiche: Sport und Politik. } \\
\text { Sie scheinen auf dem ersten Blick widersprüchlich } \\
\text { zu sein. Die Äußerung wird klar, wenn sie in } \\
\text { der politischen Umgebung gesagt wird. „Ein } \\
\text { Schaukampf“ gehört zum Sportbereich und bedeutet } \\
\text { einen Boxkampf, bei dem es nicht um einen } \\
\text { Wettbewerb geht. Das Wort wird in die Politikwelt } \\
\text { übertragen, wo die Politiker, wenn sie in der Wahl } \\
\text { gewinnen wollen, einen Kampf gegen andere } \\
\text { führen. Die Metapher dient zur Übertreibung und } \\
\text { Veranschaulichung. }\end{array}$ \\
\hline & $\frac{\frac{\text { Substantivische }}{\text { Gruppe (oder }}}{\frac{\text { Substantiv) }}{\text { Verb }}+}$ & $\begin{array}{c}\begin{array}{c}\text { Bundespräsident } \\
\text { kann keine } \\
\text { Schleiflackfigur sein }\end{array} \\
\end{array}$ & $\begin{array}{l}\text { Bereich des Handwerks. } \\
\text { Er muss wirken, arbeiten. Die Widersprüchlichkeit } \\
\text { der Substantive sieht man aus den unterschiedlichen } \\
\text { Lebensbereichen. „Der Schleiflack“ gehört zum } \\
\text { Handwerkbereich, mit ihm werden die hölzerne } \\
\text { Figuren oder Möbel lackiert und geschliffen., ,Figur“ } \\
\text { ist hier eine politische Figur, der Bundespräsident } \\
\text { gemeint. Anstatt direkt zu sagen, dass der } \\
\text { Bundespräsident keine Wirkung zeigt und nichts tut, } \\
\text { benutzt man die Metapher, „Schleiflackfigur“, Figur, } \\
\text { die nur zum Anschauen dasteht. In dieser Metapher } \\
\text { steckt Ironie und negative Bewertung durch den } \\
\text { Sprecher. }\end{array}$ \\
\hline & $\frac{\frac{\text { Substantivische }}{\text { Gruppe (oder }}}{\frac{\text { Substantiv) }}{\text { Adjektiv }}+}$ & $\begin{array}{l}\text { dieselben politischen } \\
\text { Krähen }\end{array}$ & $\begin{array}{l}\text { Tierbereich. } \\
\text { Politiker, die viel schreien und nichts machen. } \\
\text { Die bildhafte Metapher besteht aus zwei } \\
\text { Lebensbereichen: Politik- und Tierbereich „Krähen“. } \\
\text { Die Krähe ist ein schwarzer Vogel, die viel kräht, } \\
\text { aber diese Laute sind unangenehm. Die Krähen sind } \\
\text { von Menschen nicht besonders beliebt. „Dieselben } \\
\text { politischen Krähen“ sind solche Politiker, die } \\
\text { negativ bewertet werden, weil sie viel sprechen, aber } \\
\text { wenig machen. }\end{array}$ \\
\hline 2. & $\begin{array}{l}\text { Adjektivische } \\
\text { Gruppe }\end{array}$ & & \\
\hline & $\frac{\text { Adjektiv }}{\text { Substantiv }}$ & $\begin{array}{l}\text { in einer sterilen } \\
\text { großen Koalition }\end{array}$ & $\begin{array}{l}\text { Medizinbereich. } \\
\text { In einer wirkungslosen, unproduktiven Großen } \\
\text { Koalition. } \\
\text { In der adjektivischen Metapher werden Wörter } \\
\text { aus gegensätzlichen Bereichen: Medizinbereich } \\
\text { „sterilen“ und Politikbereich „Großen Koalition“ } \\
\text { miteinander verknüpft. In dem direkten Wortbereich } \\
\text { bedeutet „steril““ „unfruchtbar“. Die Übertragung } \\
\text { des Wortes in den Politikbereich schafft eine } \\
\text { neue, metaphorische Bedeutung: die Koalition } \\
\text { ist unproduktiv, nicht wirksam. Sie wirkt negativ, } \\
\text { bewertend. }\end{array}$ \\
\hline
\end{tabular}




\begin{tabular}{|c|c|c|c|}
\hline & $\begin{array}{c}\text { Substantivische } \\
\text { Gruppe }+ \\
\text { Adjektiv }\end{array}$ & $\frac{\text { das frostige }}{\text { Verhältnis zu China }}$ & $\begin{array}{l}\text { Naturbereich. } \\
\text { Unfreundliches, distanziertes Verhältnis. Diese } \\
\text { Metapher verbindet zwei Bereiche: Natur- ,frostige“ } \\
\text { und Menschenbeziehungsbereich, „Verhältnis“. Die } \\
\text { Metapher informiert über Verhältnisse zwischen den } \\
\text { Ländern. Das Wort „,frostig“ versteht man als etwas } \\
\text { Kaltes, Negatives. Deswegen ist das Verhältnis } \\
\text { als unfreundlich, distanziert zu verstehen. Diese } \\
\text { Metapher gibt dem Text durch das Gefühl der Kälte } \\
\text { die Bedeutung. Sie wirkt übertreibend. }\end{array}$ \\
\hline 3. & Verbale Gruppe & & \\
\hline & $\begin{array}{c}\text { Substantivische } \\
\text { Gruppe (oder } \\
\text { Substantiv) }+ \\
\underline{\text { Verb }}\end{array}$ & Theorien $\underline{\text { schmieden }}$ & $\begin{array}{l}\text { Bereich des Handwerks. } \\
\text { Viel und immer nur sprechen und nichts machen. } \\
\text { Die Metapher besteht aus verschiedenen Bereichen: } \\
\text { Politik, „Theorien“ und Handwerkbereich } \\
\text { „schmieden“. Die normale Bedeutung des Verbs } \\
\text { ist „mit einem scharfen Werkzeug arbeiten“. Im } \\
\text { politischen Zusammenhang bekommt das Wort eine } \\
\text { neue Bedeutung und es heißt, dass die Politiker nur } \\
\text { sprechen und nicht praktisch etwas machen. Die } \\
\text { Metapher charakterisiert, bewertet negativ. }\end{array}$ \\
\hline & $\begin{array}{c}\text { Substantivische } \\
\text { Gruppe }+ \\
\text { Infinitiv }\end{array}$ & $\begin{array}{l}\text { das Problem der } \\
\text { Osterweiterung } \\
\text { frisieren }\end{array}$ & $\begin{array}{l}\text { Schönheitsbereich. } \\
\text { Ändern, verfälschen. In der Metapher werden zwei } \\
\text { Bereiche zusammengesetzt: Politik ,,das Problem } \\
\text { der Osterweiterung“" und Lebensbereich „frisieren“. } \\
\text { Das Verb „,frisieren“ assoziieren wir mit einem } \\
\text { Friseur, der das Haar schön herrichtet und auch } \\
\text { unser Aussehen ändert. Die Übertragung des Wortes } \\
\text { in die Politik schafft einen neue Bedeutung: das } \\
\text { Problem, die Frage wird verfälscht. }\end{array}$ \\
\hline & $\begin{array}{c}\text { Substantivische } \\
\text { Gruppe }+ \\
\underline{\text { zu }+ \text { Infinitiv }}\end{array}$ & $\begin{array}{l}\text { Gesprächschancen } \\
\text { zu torpedieren }\end{array}$ & $\begin{array}{l}\text { Kriegsbereich. } \\
\text { In gezielter Weise stören. Die Metapher verbindet } \\
\text { zwei Bereiche - Lebens- „Gesprächschancen“" und } \\
\text { Militär- oder Kriegsbereich „torpedieren“. Das Wort } \\
\text { „torpedieren“ bedeutet etwas ,verhindern“, „,stören“ } \\
\text { und wirkt im politischen und wirtschaftlichen } \\
\text { Bereich als abwertend }\end{array}$ \\
\hline
\end{tabular}

Substantivische Metaphern. Substantivische Metaphern bilden die größte Zahl aller Beispiele. Die Funktion der substantivischen Metaphern ist die Information an den Empfänger effektvoller zu bringen. Diese Metaphern haben besonders große Wirkung, betonen die gewünschte Auskunft und Ansicht des Senders und so lenken die Aufmerksamkeit des Hörers/Lesers.

Adjektivische Metaphern. In den Interviewtexten werden auch adjektivische Metaphern benutzt, obwohl sie nicht so oft vorkommen. Sie ist die kleinste Gruppe der Beispiele. Die adjektivischen Metaphern werden meistens mit Substantiven benutzt. Sie bezeichnen Eigenschaften und dadurch bekommt die Aussage eine neue Wirkungskraft. Die adjektivischen Metaphern charakterisieren eine Person 
oder Sache, geben einen neuen Inhalt und machen das Gesagte informationsreicher und verständlicher.

Verbale Metaphern. Sie sind in der Pressesprache oft zu treffen. Die metaphorisch gebrauchten Verben bedeuten aktives Einwirken auf die Umwelt und menschliches Handeln (erobern, parken, hinterherhinken, frisieren usw.). Sie werden an ein Subjekt gebunden, das zu diesem Handeln unfähig ist. So wird der Leser/Hörer gezwungen, dem Satz eine übertragene, vom normalen Textverständnis abweichende Bedeutung zu geben.

Die Textsorte, die untersucht wurde, ist das Interview in der Politik und Wirtschaft. Die Antworten der Politiker und Wirtschaftsleute sind nicht lang, aber durch Metapherngebrauch sehr informativ und effektiv. In ihrer Sprache widerspiegelt sich meistens Bewertung und Übertreibung.

\section{Forschungsergebnisse}

In der Zeitschrift „Der Spiegel“ wurden Texte aus folgenden Bereichen analysiert: Wahlkampf- (22), Geld- (20), Auslands- (19) und Reformpolitik (15), Umwelt- (7), Arbeits- (6), Menschenrechte (5), Welt- (4) und Finanzenwirtschaft (10), Elektronik (2), Auto- (4), Mechanik- (2) und Medienindustrie (3).

Insgesamt wurden 309 Metaphernbeispiele gesammelt. Die Metaphern wurden nach kommunikativen und lexikologischen Merkmalen klassifiziert. Die größte Zahl der Metaphern bildet die substantivische Gruppe.

\section{Resultate:}

- In der linguistischen Strukturforschung und Stilistik bezeichnet man die Metapher als eine der wichtigsten von allen Tropen und Figuren, so dass die Metaphernforschung heute einen eigenen Spezialforschungsbereich ausmacht.

- Die Menschen wurden von der Zeitschrift „Der Spiegel“ meistens über Wahlkampf-, Geld-, und Reformpolitik interviewt. Im Interview, in der Sprache der Politiker und Wirtschaftsleute wiederspiegelt sich meistens Bewertung und Übertreibung.

- Die größteZahl der Bereiche bildeten die Metaphern zum Wahlkampfthema (13): Kriegs-, Natur-, Theater-, Verkehrs-, Maschinerie-, Sport-, Musik-, Tier-, Medizin-, Mystik-, Essens-, Lebens-, Handwerksmetaphern. Die kleinste Zahl der metaphorischen Bereiche waren in den Interviews von Umwelt- und Arbeitspolitik zu finden (5 Bereiche). Die häufigsten Bereiche in analysierten Beispielen: Lebens-, Kriegs,-, Tier, -Sport-, und Theaterbereich. Für alle Themen ist die Kriegsmetaphorik typisch, außer „Menschenrechte“ und „Umweltpolitik“. 


\section{Literaturverzeichnis}

1. Bertau, M. C. (2013). Sprachspiel Metapher: Denkweisen und kommunikative Funktion einer rhetorischen Figur. Springer-Verlag.

2. Bertau, M. C. (1999). Spuren des Gesprächs in innerer Sprache. Versuch einer Analyse der dialogischen. Zeitschrift für Sprache \& Kognition, 18(1/2), 4-19.

3. Bußmann, H., \& Lauffer, H. (2008). Lexikon der Sprachwissenschaft.

4. Davidson, D. (1998). Was Metaphern bedeuten. Die paradoxe Metapher, Frankfurt am Main, 49, 75.

5. Debatin,B.(1995).DieRationalitätderMetapher:einesprachphilosophische und kommunikationstheoretische Untersuchung. Walter de Gruyter.

6. Engel, U. (1991). Deutsche Grammatik. 2., verb. Aufl. Heidelberg: Groos.

7. Frieling, G. (1996). Untersuchungen Zur Theorie der Metapher Das Metaphern-Verstehen Als Sprachlich-Kognitiver Verarbeitungsprozess.

8. Glück, H. (Ed.). (2016). Metzler Lexikon Sprache. Springer-Verlag.

9. Haller, M (2001). Das Interview. Ein Handbuch für Journalisten, UVK Verlagsgesellschaft mbH, Konstanz.

10. Karaliūnas, S. (1997). Kalba ir visuomenè. Psichosociologiniai ir komunikaciniai kalbos vartojimo bruožai.

11. Kirchhoff, S. (2015). Krieg mit Metaphern: Mediendiskurse über 9/11 und den» War on Terror «(Vol. 2). transcript Verlag.

12. Kohl, K. (2007). Metapher. In Metapher (pp. 19-72). JB Metzler.

13. Laser, B. (2005). Die kognitive Metapher als Werkzeug des Denkens. Zur Rolle der Analogie bei der Gewinnung und Vermittlung wissenschaftlicher Erkenntnisse. Informationen Deutsch als Fremdsprache, 32(2-3), 144-146.

14. Lau, D. (2006). Metapherntheorien der Antike und ihre philosophischen Prinzipien. Ein Beitrag zur Grundlagenforschung in der Literaturwissenschaft. Frankfurt (Main) ua.

15. Schmitt, C. (1988). Gemeinsprache und Fachsprache im heutigen Französisch. Formen und Funktionen der Metaphorik in wirtschaftsfachsprachlichen Texten. Fachsprachen in der Romania, Tübingen, 113-129.

16. Schneider, J. (2007). Metapherntheorien und Übersetzung.

17. Strauch, D., \& Rehm, M. (2007). Lexikon Buch-Bibliothek-neue Medien. Walter de Gruyter.

18. Sowinski, Bernhard, Stil, in: Gert Ueding/Gregor Kalivoda (ed.), Historisches Wörterbuch der Rhetorik, Vol. 8, Darmstadt 2007, 1393-1419).

19. https://www.duden.de (žr.: $2018 \mathrm{~m}$. sausio mèn.) 


\title{
KOMUNIKACINĖS IR KOGNITYVINĖS METAFORŲ \\ FUNKCIJOS PUBLICISTIKOS TEKSTE
}

\author{
Lekt. Jurgita Lukienè \\ Kauno technologijos universitetas \\ Doc. dr. Nijolė Čiučiulkienė \\ Aleksandro Stulginskio universitetas
}

Dainoras Lukas

žurnalistas, sociologas

\section{Santrauka}

Šiame straipsnyje nagrinejjamos komunikacinès ir kognityvinès metaforu funkcijos interviu tekste. Svarbus šios temos klausimas - ką mums duoda metafora? Šios stiliaus figūros funkcija ịvairiais laikotarpiais buvo suvokiama vis kitaip. Šiandien metafora vartojama labai plačiai. Ji dažna ne tik literatūroje, bet ir techninèje kalboje, spaudoje. Metaforos lyginamos su galvosūkiais. Suprasti metaforos reikšmę - tai tas pats, kas iššifruoti kodą. Kalbètojo tikslas - ne tam tikra metaforos reikšmè, o jos funkcija. Ji padeda pamatyti ir pažinti kažką nauja. Šiuolaikinè kalba geriausiai atsispindi laikraščiuose ir žurnaluose. Čia svarbus tampa žodžių parinkimas, sakinio struktūra, tema, situacija. Todèl praktinèje dalyje nagrinètas vokiečiu politikos ir ekonomikos žurnalas „Der Spiegel“. Nagrinèti tekstai šiomis temomis: pinigai, rinkimai, aplinkosauga, darbas, užsienis, reformos, žmonių teisès, pasaulis, finansai, elektronika, automobiliai, mechanika, medijos. Iš viso iš įvairių žurnalo interviu surinkti 309 metaforų pavyzdžiai. Jie analizuoti substitucijos būdu. Tirta ne tik metaforos reikšmé, bet ir kalbėtojo tikslas, metaforos poveikis. Politikos ir ekonomikos ekspertų kalboje metaforų vartojimas yra labai informatyvus ir efektyvus. Jų kalboje atsiskleidžia vertinimas ir situacijos perdetas sureikšminimas. Metaforų poveikis yra skirtingas, dažnai ji galima suprasti tik iš konteksto. Jos apibūdina, informuoja, išreiškia vertinimą, taip darydamos stiprų poveikị skaitytojui. Tirtuose pavyzdžiuose daugiausia yra karo, gyvūnų, sporto ir teatro metaforų.

Pagrindiniai žodžiai: metafora, politika ir ekonomika, interviu. 


\section{AUTORIŲ LYDRAŠTIS}

Autoriaus vardas, pavardè: Jurgita Lukienè

Mokslo laipsnis ir vardas: -

Darbo vieta ir pareigos: Kauno technologijos universitetas, lektore

Autoriaus mokslinių interesų sritys: pedagogika, lingvistika

El. paštas: jurgita.lukiene@ktu.lt

Autoriaus vardas, pavardė: Nijolè Čiučiulkienè

Mokslo laipsnis ir vardas: socialinių mokslų daktarè, docentė

Darbo vieta ir pareigos: Aleksandro Stulginskio universitetas, Filosofijos, psichologijos ir profesinès edukologijos katedra

Autoriaus mokslinių interesų sritys: komunikavimo emancipavimas, mokytojų rengimas, mentorysté/ reversinè mentoryste, probleminis mokymasis (PBL).

El. paštas: nijole.ciuciulkiene@asu.lt

Autoriaus vardas, pavardè: Dainoras Lukas

Mokslo laipsnis ir vardas: -

Darbo vieta ir pareigos: Viešoji įstaiga Žemès ūkio konsultavimo tarnyba, žurnalas „Mano ūkis“, redaktorius

Autoriaus mokslinių interesų sritys: sociologija, politika, miestų ir politikų jivaizdžiai

El. paštas: lukasdainoras@yahoo.com

\section{AUTHORS' COVER LETTERS}

Author's name and surname: Jurgita Lukienè

Academic degree and name:

Workplace and position: Kaunas University of Technology, Department of Intercultural Communication and Modern Languages; Lecturer

Author's research interests: pedagogy, linguistics

E-mail address: jurgita.lukiene@ktu.lt

Author's name and surname: Nijolè Čiučiulkienė

Academic degree and name: Doctor of Social Sciences

Workplace and position: Aleksandras Stulginskis University, Department of Philosophy, Psychology and Vocational Education, Associate Professor

Author's research interests: emancipation of communicating, teacher training, mentoring/reverse mentoring, problem-based learning

E-mail address: nijole.ciuciulkiene@asu.lt 
Author's name and surname: Dainoras Lukas

Academic degree and name:

Workplace and position: agribusiness magazine Mano ūkis, Editor, Lithuanian Agriculture Advisory Service

Author's research interests: sociology, politics, images of cities and politicians

E-mail address: lukasdainoras@yahoo.com 